\title{
Comparison of antioxidants content and activity of Nephelium mutabile rind extracted using ethanol and water
}

\begin{abstract}
This study is aimed to determine and compare the effect of different solvent polarities on total phenolic content (TPC), total flavonoid content (TFC), and antioxidant activities using water and ratio of aqueous/ethanol (40, 60, and 80\%) for the extraction of Nephelium mutabile rind. The TPC and TFC were evaluated using Folin-Ciocalteu reagent and aluminum chloride colorimetric method, respectively; while antioxidant activities were determined by DPPH radical scavenging and FRAP assays. The highest TPC was determined in the water extract of $\mathrm{N}$. mutabile rind, followed by $80 \%$ ethanol extract. The highest TFC was found in $60 \%$ ethanol extract of $\mathrm{N}$. mutabile rind, followed by $80 \%$ ethanol extract. Similar to the TPC, the N. mutabile rind extract exhibited the highest FRAP value when extracted with water, followed by $80 \%$ ethanol extract. However, the antioxidant activity assessed by DPPH radical scavenging assay was the highest in $80 \%$ ethanol extract of $\mathrm{N}$. mutabile rind, followed by water extract. A strong and positive correlation $(r=0.655)$ was found between TPC and antioxidant activities of the N. mutabile rind extracts. The result obtained indicates that the high antioxidant activities of N. mutabile rind are probably due to the high TPC in the extract. The results from this study indicated that water could be a useful solvent in extracting antioxidant compounds from the N. mutabile rind. In future, the water and $80 \%$ ethanol extracts of N. mutabile rind could be used as nutraceuticals.
\end{abstract}

Keyword: DPPH; Flavonoid; FRAP; Pulasan; Phenolic 